\title{
Extended Jets of AGN: Proton Synchrotron Emission
}

\section{Partha Pratim Basumallick and Nayantara Gupta*}

Astronomy \& Astrophysics Group

Raman Research Institute

C.V.Raman Aveneu, Sadashivanagar

Bangalore 560080, India

E-mail: nayan@rri.res.in

\begin{abstract}
Many extended jets of kilo-parsec scale size from Active Galactic Nuclei have been detected by Hubble and Chandra telescopes. IC/CMB model for X-ray emission from six extended jets has been ruled out by radio data from ALMA observations and also the predicted gamma ray flux in this model overshot the Fermi LAT upper limits. We have selected the sources 3C 273, PKS 0637-752, B3 0727+409 and AP Librae for our study. We show the proton synchrotron model may explain the X-ray emission in some cases without violating luminosity constraints. The kiloparsec scale jet could be moving with Doppler factor close to 1 in this scenario unlike the IC/CMB model which requires higher values of Doppler factor to explain the observed emission.
\end{abstract}

7th Fermi Symposium 2017

15-20 October 2017

Garmisch-Partenkirchen, Germany

\footnotetext{
*Speaker.
} 


\section{Introduction}

Our work is motivated by a series of papers which rule out IC/CMB model from six extended jets of Active Galactic Nuclei $[1,2]$. Proton synchrotron emission from extended jets of Flat Spectrum Radio Quasars (FSRQs) has been studied earlier for Pictor A, 3C 120, 3C 273, PKS 0637-752 in [3], for 3C 273 in [4] and for PKS 0637-752 in [5]. The age of the extended jet is assumed to be of the order of $10^{8}$ years, lower than the typical age of AGN. The ultrahigh energy protons are trapped in the kpc scale jet of $\mathrm{mG}$ scale magnetic field for $10^{7}-10^{8}$ years which is comparable to the synchrotron radiation loss time scale of the protons. Within this scenario the extended jet has a bulk Lorentz factor in the range of 1-3 and the escape time of the protons are assumed to be energy dependent. The authors ([3], [4], [5]) have compared the synchrotron loss and escape time scales of the ultrahigh energy protons with the age of the extended jet to determine the break energy in the proton spectrum. Finally they obtained the high energy photon flux from the synchrotron emission of the trapped protons.

The possibility of Bethe-Heitler pair and photo-meson production in extended jet of PKS 0637752 has been discussed in [6]. The authors have used magnetic field of the order of $0.1-0.01 \mathrm{mG}$ (which makes proton synchrotron emission insignificant in their model) and an energy independent escape time of $10^{4}$ years for the protons. The authors have argued that the age of the extended jet has to be comparable to the light crossing time, which is $10^{3}-10^{4}$ years. It is difficult to physically realise this scenario as the protons are trapped in the magnetic field of the extended jet and can not escape at the speed of light. The proton luminosity required in the model discussed in [6] is $10^{49}$ $\mathrm{erg} / \mathrm{sec}$ which is 10-100 times the Eddington's luminosity of PKS 0637-752.

We have revisited the possibility of proton synchrotron emission from the extended jets of $3 \mathrm{C}$ 273, PKS 0637-752, B3 0727+409.

Moreover the multi-wavelength data compiled in [7] has been used to study the possibility of proton synchrotron origin of very high energy gamma ray emission from the extended jet of AP Librae. We have considered a three zone model with a compact emission region or blob, a parsec scale jet and a kilo-parsec scale extended jet.

\section{Modeling and Results}

Assuming a simple power law proton spectrum of spectral index 2 in the energy range of $10^{15}$ $10^{18} \mathrm{eV}$ we have calculated the photon fluxes for the extended jets of 3C 273, PKS 0637-752 and B3 0727+409 to explain the X-ray data with the proton synchrotron model. The emission region is assumed to be of radius $1 \mathrm{kpc}$ and its Doppler factor is fixed at 1 for simplicity. Figure-1, Figure-2 \& Figure-3 shows the contour plots for the extended jets of these three sources for magnetic field within $15 \mathrm{mG}$. We have also assumed equipartition in energy between the magnetic field and the relativistic protons. The jet power required to explain the observed X-ray data is $\sim 1.28 \times 10^{48}$ ergs/sec (corresponding to $4.25 \mathrm{mG}$ ) for $3 \mathrm{C} 273, \sim 3 \times 10^{48} \mathrm{ergs} / \mathrm{sec}$ for knot wk8.9 (corresponding to $6.5 \mathrm{mG}) \& \sim 5.75 \times 10^{48} \mathrm{ergs} / \mathrm{sec}$ for the brightest knot (corresponding to $9 \mathrm{mG}$ ) of PKS 0637 752. The Eddington's luminosity of 3C 273 and PKS $0637-752$ could be as high as $10^{48} \mathrm{erg} / \mathrm{sec}$ ([8], [6]). Thus the jet powers required to explain the X-ray data from the extended jets of $3 \mathrm{C}$ 273 (for magnetic field $4.25 \mathrm{mG}$ ) and PKS 0637-752 (for magnetic fields of $6.5 \mathrm{mG} \mathrm{\&} 9 \mathrm{mG}$ ) are 
comparable to their Eddington's luminosities. Hence it is possible that proton synchrotron emission could be the underlying mechanism of X-ray emission from their extended jets. B3 0727+409 is located at a redshift of 2.5 having a black hole mass of $3.3 \times 10^{8}$ solar mass ([9]), Eddington's luminosity $4 \times 10^{46} \mathrm{ergs} / \mathrm{sec}$. In Figure- 3 the butterfly shows the possible X-ray emission from the extended jet of this source. In this case the jet power required to explain the X-ray emission with proton synchrotron model is $7.1 \times 10^{48} \mathrm{ergs} / \mathrm{sec}$ (corresponding $10 \mathrm{mG}$ ), which exceeds the Eddington's luminsoity of this source.

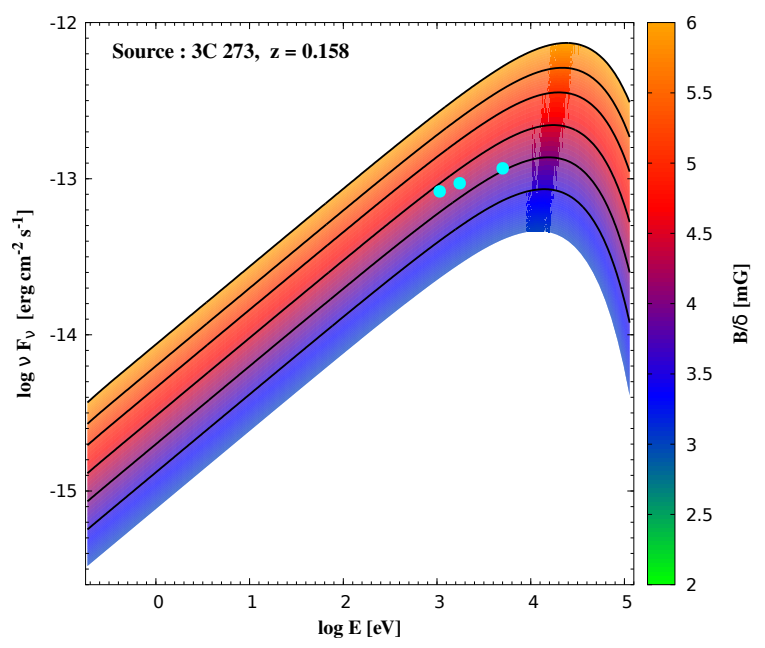

Figure 1: Photon flux from Proton synchrotron emission vs Energy (in logscale) contour plot assuming equipartition of energy in protons and magnetic field. The contour lines are for specific values of the magnetic field $B$ which is equivalent to $\frac{B}{\delta}$ (as $\delta=1$ for these plots). The contour lines range from $\frac{B}{\delta}=3.5 \mathrm{mG}-6 \mathrm{mG}$ in steps of $500 \mu \mathrm{G}$. X-ray data poins are shown with cyan dots.

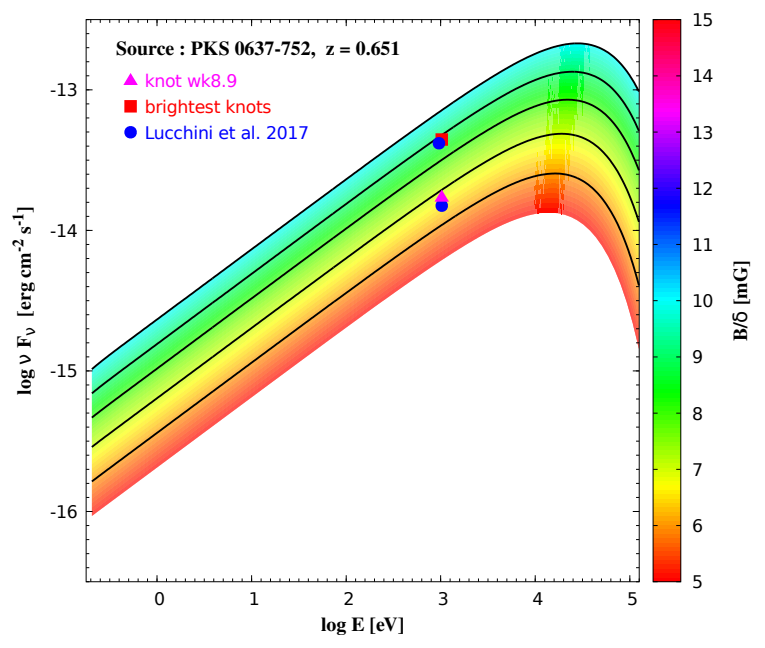

Figure 2: Same as in Figure-1 with the source as PKS 0637-752. The contour lines range from $\frac{B}{\delta}=6 \mathrm{mG}-10 \mathrm{mG}$ in steps of $1 \mathrm{mG}$. X-ray data points are shown. The blue points are taken from [10] blue dots, red square and pink triangle.

The modeling has been done also for AP Librae with power law spectrum of injected particles (electrons and protons) in the extended jets. The cooling break energy has been calculated and the propagated or steady state spectrum of particles has been used to calculate the photon spectrum 


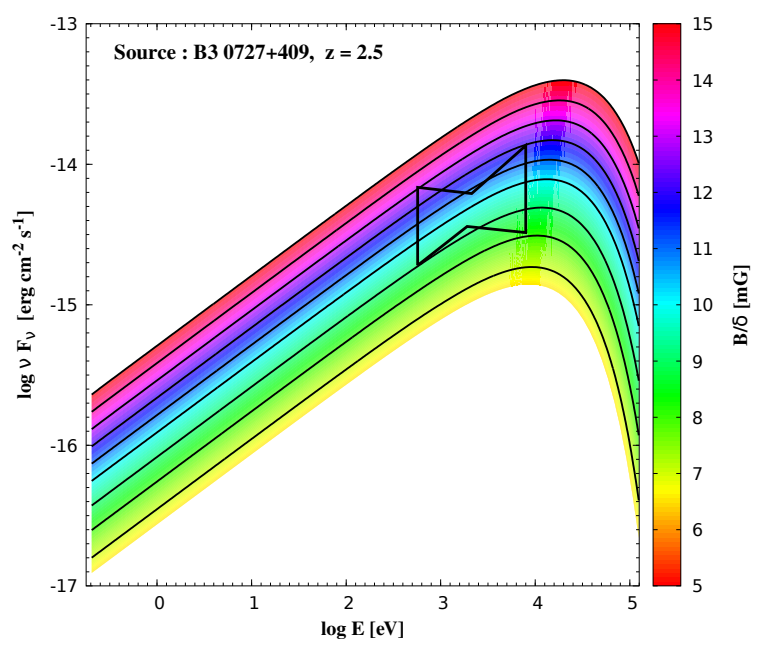

Figure 3: Same as in Figure-1 with the source as $B 30727+409$. The contour lines range from $\frac{B}{\delta}=7 \mathrm{mG}-15 \mathrm{mG}$ in steps of $1 \mathrm{mG}$. Butterfly contour shows the X-ray flux.

from radiative losses. We have considered three zones inside AP Librae to explain the entire spectral energy distribution of this source. Zone-1 is a blob near the core, Zone-2 is a near parsec scale jet and Zone-3 is the kilo-parsec scale extended jet. The electron spectra in Zone-1 and Zone-2 are broken power laws as they are cooling fast by synchrotron and self Compton emission.

$$
\frac{d N_{e l}\left(E_{e l}\right)}{d E_{e l}}=A \begin{cases}E_{e l}^{-p_{1}} & E_{e l}<E_{e l}^{b r k} \\ E_{e l}^{b r k} E_{e l}^{-p_{1}-1} & E_{e l}>E_{e l}^{b r k}\end{cases}
$$

Out of the several input parameters required by the code, the extrema of the relativistic particles along with the so called break energy $E_{e l}^{b r k}$ are a prime factor in determining the generated spectrum. The break energy is determined by equating the cooling time $t_{\text {cool }}$ with $t_{e s c}=\eta_{e s c} \times \frac{R}{c}$, where $\frac{R}{c}$ is the light travel time in the emission region and $\eta_{\text {esc }}$ acts as a scaling factor. The cooling time scale of the electrons is given by the expression

$$
\frac{1}{t_{\text {cool }}}=\frac{1}{t_{\text {synch }}}+\frac{1}{t_{\text {SSC }}}
$$

which is a convolution of both the synchrotron cooling and SSC cooling timescales of the electrons. In a more detailed form Eq.2.2 can be expressed as follows

$$
\frac{1}{t_{c o o l}}=\frac{4}{3} \sigma_{T h}^{e l} \beta_{e l}^{2} \gamma_{e l} \frac{c}{m_{e l} c^{2}}\left(U_{B}+U_{e l}^{\text {synch }}\right)
$$

where $\sigma_{T h}^{e l}$ is the Thomson cross-section of electrons. The dimensionless speed of electrons $\beta_{e l} \simeq 1$ and Lorentz factor $\gamma_{e l}=\frac{E_{e l}}{m_{e l} l^{2}} . U_{B}\left(=\frac{B^{2}}{8 \pi}\right)$ and $U_{e l}^{\text {synch }}$ are the energy densities ${ }^{1}$ of the magnetic field and the synchrotron radiation generated by the electrons respectively. The KleinNishina effect does not play any significant role in our calculations. In our model the radius of the central core region is assumed to be $R_{Z 1}=8.65 \times 10^{16} \mathrm{~cm}$ while the radius of the parsec scale region

\footnotetext{
${ }^{1}$ All the expressions \& values of energy densities are in the comoving frame of the emission regions throughout the paper unless otherwise mentioned.
} 
is taken as $R_{Z 2}=9.5 \times 10^{17} \mathrm{~cm}$. The magnetic field in the two zones are respectively $B_{Z 1}=8.5$ $\mathrm{mG}$ and $B_{Z 1}=1.72 \mathrm{mG}$. The scaling factor $\eta_{\text {esc }}$ for Zone-1 and Zone-2 are respectively 62 and 63 . As the value of $U_{e l}^{\text {synch }}$ is sensitive to magnetic field, the energy range and also the energy density of the particle population, we have to adjust our parameter values before we can fix the break energy of the electrons for a suitable choice of $\eta_{\text {esc }}$. The relativistic protons are cooled by synchrotron emission inside the blob. The synchrotron emission from relativistic protons inside Zone-1 and Zone-2 is generated by assuming that the energy density ratio between protons and electrons is 1000:1. Proton cooling is not important in these zones and as the cooling time scale is long no break appears in the proton spectrum. The maximum energy of the protons in the emission region is constrained by the condition:

$$
B \geqslant 30 \frac{E_{p}^{\max }(\mathrm{eV})}{10^{19}} \frac{10^{15}}{R(\mathrm{~cm})} \quad \text { in Gauss. }
$$

which ensures that for our choice of $B$ the Larmor radii of protons having energy $E_{p}^{\max }$ (in $\mathbf{e V}$ ) does not exceed the chosen value of $R(\mathbf{i n} \mathbf{~ c m})$. It is important to mention in this context that although AP Librae exhibits intra-day scale (sometimes even as small as $20 \mathrm{~min}$ ) variability in the optical frequency range, we do not consider it as a constraining factor of $R$ owing to the fact that the data being modeled represents a flux averaged state of the source. However according to the approximate relation

$$
R \leq \frac{c \Delta t_{o b s} \delta}{1+z}
$$

a variability timescale $\left(\Delta t_{o b s}\right)$ of 1 day for the Doppler factor $\delta=\frac{1}{\Gamma(1-\beta \cos \theta)}=12.3$ gives the radius of the emission region as $\sim 3 \times 10^{16} \mathrm{~cm}$. Similarly for the Fermi-LAT observed 6 day variability observed in frequencies above $300 \mathrm{MeV}$ the radius of the emission region should be $\sim 2 \times 10^{17}$ $\mathrm{cm}$. However taking into account the fact that 2.5 might lead to large errors while estimating the dimensions of the emitting region our estimate of $R_{Z 1}=8.65 \times 10^{16} \mathrm{~cm}$ and $R_{Z 2}=9.5 \times 10^{17}$ $\mathrm{cm}$ should be admissible even when modelling the optical frequency data with day/intra-day scale variability and the HE $\gamma$-ray frequency data with week scale variability.

\subsection{Particle Spectra in Extended Jet}

The electron spectrum in the extended jet (Zone-3) also follows a broken power law given by Eq.2.1. The electron population in the extended jet radiates primarily via synchrotron emission (unlike the blob where SSC cooling is also a major contributor). The break energy of electrons $E_{b r k, j e t}^{e l}$ is calculated by equating the synchrotron cooling time $\left(t_{\text {synch }}^{e l}\right)$ with the extended jet lifetime $\left(t_{j e t}\right)$.

$$
t_{\text {synch }}^{e l}=t_{j e t} \Rightarrow \frac{\gamma_{e l} m_{e l} c^{2}}{\frac{4}{3} \sigma_{T h}^{e l} c U_{B} \gamma_{e l}^{2} \beta_{e l}^{2}}=t_{j e t}
$$

The age of the extended jet $t_{j e t}$ is assumed to be of the order of $10^{5}-10^{7}$ yrs. We note that the justification for assuming this age is to allow the ultrahigh energy protons to cool down by synchrotron emission during the lifetime of the jet.

The proton synchrotron emission from the extended jet of AP Librae is considered as the origin of the HE-VHE $\gamma$-rays in our model. A broken power law proton spectrum is required to fit the $\gamma$ - 
ray data.

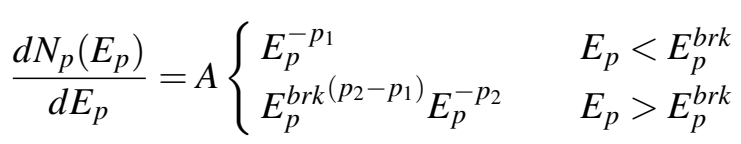

We consider energy dependent diffusive escape of particles from the kpc scale extended jet and not the much smaller Doppler boosted compact regions because the particles escape from the compact regions at a much faster rate, implying no significant amount of diffusion.

We compare the synchrotron cooling time with the age of the extended jet and the escape timescale of the protons to fix the break energy. The resulting proton spectrum is characterized by a change in the spectral index from $p_{1}$ before the break to $p_{2}$ after the break, in Bohm diffusion limit $p_{2}=p_{1}+1$. Also as the particles, both electrons and protons, are accelerated in the same region whether it be the blob or the extended jet, the spectral indices for both particle populations $\left(p_{1}\right)$ before cooling, have been kept the same for the specific emission regions. The maximum energy of the protons on the other hand are constrained by the condition in Eq.2.4. The minimum energy of the protons is a free parameter and set to values of the order $\sim 10^{16}-10^{17} \mathrm{eV}$. Although the minimum energy values are a consequence of the modelling requirements of the observed SED, higher values of the minimum energy reduce the jet power appreciably.

The escape timescale and the synchrotron cooling time of the protons are given by Eq.2.8 \& Eq.2.9.

$$
\begin{gathered}
t_{\text {esc }, \text { Bohm }} \simeq 4.2 \times 10^{5} \eta^{-1} B_{\mathrm{mG}} R_{\mathrm{kpc}}^{2}\left(E_{p} / 10^{19} \mathrm{eV}\right)^{-1} \mathrm{yrs} \\
t_{\text {synch }} \simeq 1.4 \times 10^{7} B_{\mathrm{mG}}^{-2}\left(E_{p} / 10^{19} \mathrm{eV}\right)^{-1} \mathrm{yrs}
\end{gathered}
$$

In Eq.2.8, $\eta$ is the gyrofactor which assumes the value of 1 in the Bohm diffusion limit. $B_{\mathrm{mG}}$ is the ambient magnetic field (expressed in $\mathrm{mG}$ units) in the extended jet and $R_{\mathrm{kpc}}=\frac{R}{3.08 \times 10^{21}}$ where $R$ is the radius of the emission zone (expressed in cm units) in the extended jet. When modelling the data in the Bohm diffusion regime we assume the value of $t_{j e t}=2 \times 10^{7} \mathrm{yrs}$. In our model in Bohm diffusion regime $E_{p}^{b r k}=2.51 \times 10^{18} \mathrm{eV}$ for which the values of $t_{e s c} \& t_{\text {synch }}$ are $2.53 \times 10^{7}$ yrs \& $5.6 \times 10^{7}$ yrs respectively, which are comparable to the extended jet lifetime of $2 \times 10^{7} \mathrm{yrs}$.

We also consider the Kolmogorov and Kraichnan diffusion regimes to study the proton synchrotron model, the diffusion time scale is given in Eq.2.10

$$
t_{\text {esc }} \simeq \frac{R}{c}\left(\frac{E_{p}}{E_{\text {free }}}\right)^{-\alpha}
$$

where $E_{\text {free }}=E^{*} B_{4} R_{14}$ with $E^{*}=3 \times 10^{20} \mathrm{eV}, B_{4}=\frac{B}{10^{4}}$ Gauss and $R_{14}=\frac{R}{10^{14}} \mathrm{~cm}$ respectively and $\alpha=\frac{1}{3} \& \frac{3}{5}$ for Kolmogorov and Kraichnan diffusion timescales. Spectral indices in Kolmogorov model are $p_{1, \text { Kol }} \& p_{2, K o l}=p_{1, \text { Kol }}+\frac{1}{3}$ and in Kraichnan model $p_{1, \mathrm{Kra}} \& p_{2, \mathrm{Kra}}=p_{1, \mathrm{Kra}}+\frac{3}{5}$. In the Kolmogorov diffusion regime the escape timescale is $2.65 \times 10^{5}$ yrs whereas that in the Kraichnan diffusion regime is $2.1 \times 10^{6}$ yrs. The break energy for the electron population is calculated assuming a jet lifetime of $2 \times 10^{5}$ yrs and $2 \times 10^{6}$ yrs for the Kolmogorov and Kraichnan diffusion regimes respectively.

Although considering the different diffusive escape timescales does not affect the energetics and the parameter values are also similar, it is worth to note that inspite of large variations in the escape timescales (which are compared with the jet lifetime to ascertain the break energy) the 
values of the other parameters are hardly affected. Moreover as there are widely different estimates of the jet lifetime $\left(\sim 10^{7}-10^{8}\right.$ yrs ([3]) \& $\sim 10^{5}$ yrs ([6]) the assumption of different diffusive escape timescales allows us to consider comparable estimates of the jet lifetime. The fact that similar parameter values are required for each case is only found as a result of our study.

If we assume energy independent escape of protons from the extended jet then the escape time is $t_{e s c}=\eta_{\text {esc }} \times\left(\frac{R}{c}\right)$. The break energy in the proton spectrum is determined in this case by equating the synchrotron time scale to the escape time scale. We find that this scenario requires a very high value of the scaling factor $\eta_{e s c}=4409$, compared to the compact regions. It is worth noticing in this context that assuming energy independent escape timescale doesn't require changing our parameter values for proton synchrotron spectrum from extended jet.

Our detailed modeling shows that the diffusion model in the extended jet has no significant impact on the spectral energy distribution and the parameter values required to fit the multi-wavelength data. However in Ap Librae the very high energy gamma ray emission from the extended jet can not be explained by proton synchrotron model as this model requires extreme proton energy, high luminosity and magnetic field strength. From some of extended jets for example 3C 273 and PKS 0637-752 the X-ray emission could be possible from proton synchrotron emission. This work has been done with the numerical code from [11].

\section{References}

[1] E. T. Meyer, P. Breiding, M. Georganopoulos et al., New ALMA and Fermi/LAT Observations of the large-scale jet of PKS 0637-752 Strengthen the Case Against the IC/CMB Model, ApJ 835 L35 (2017)

[2] P. Breiding, E. T. Meyer, M. Georganopoulos et al., Fermi Non-detections of Four X-Ray Jet Sources and Implications for the IC/CMB Mechanism, ApJ 84995 (2017)

[3] F. A. Aharonian, Proton synchrotron radiation of large-scale jets in active galactic nuclei, MNRAS 332215 (2002)

[4] E. Kundu and N. Gupta, Possible Proton Synchrotron Origin of X-Ray \& Gamma Ray Emission in Large Scale Jet of 3C 273, MNRAS 444 L16 (2014)

[5] W. Bhattacharyya and N. Gupta, Proton Synchrotron Radiation from Extended Jets of PKS 0637-752 and 3 C 273, ApJ 817121 (2016)

[6] M. Kusunose and F. Takahara, A Photo-Hadronic Model of the Large Scale Jet of PKS 0637-752, ApJ 83520 (2016)

[7] M. Zacharias and S. J. Wagner, The extended jet of AP Librae: Origin of the very high-energy gamma-ray emission?, A\&A 588 A110(2016)

[8] S. Paltani and M. Türler, The mass of the black hole in 3C 273, A \& A 435 811(2005)

[9] M. Jamrozy, L. Stawarz, V. Marchenko et al., Peculiar radio structures in the central regions of galaxy cluster Abell 585, MNRAS 4411260 (2014)

[10] M. Lucchini, F. Tavechhio and G. Ghisellini, Revisiting the EC/CMB model for extragalactic large scale jets, MNRAS 4664299 (2017)

[11] H. Krawczynski, S. B. Hughes and D. Horan, Multiwavelength Observations of Strong Flares from the TeV Blazar IES 1959+650, ApJ 601151 (2004) 